\title{
2. \\ Ueber Systeme von Curven, welche einander überall rechtwinklig durchschneiden.
}

(Von Herrn Dr. F. F. Kummer, Professor zu Breslau.)

Bekanntlich hat, wie von Leibnitz zuerst bemerkt worden ist, ein System von confocalen Kegelschnitten die merkwürdige Eigenschaft, dass alle Hyperbeln, welche es enthält, mit allen Ellipsen sich überall rechtwinklig durchschneiden. Eben so hat ein System von Parabeln, welche den Brennpunct und die Hauptaxe gemein haben, die Eigenschaft, dass alle nach der einen Richtung der Axe liegenden Parabeln mit allen nach der entgegengesetzten Richtung liegenden sich stets unter rechten Winkeln schneiden. Da mit diesen Sätzen über die confocalen Kegelschnitte viele andere sehr schöne Eigenschaften derselben zusammenhangen, so schien es mir der Mühe werth, dergleichen Systeme cinander überall rechtwinklig schneidender Curven auch für beliebige höhere Grade zu suchen. Es ist mir gelungen, die unmittelbare Quelle für dergleichen Systeme von Curven zu finden, aus welcher sich eine unendliche Anzahl derselben herleiten lässt; wie sich in dem Folgenden zeigen wird.

Es sei $f(x, y, a)=0$ die Gleichung eines Systems von Curven, in welcher $a$ ein veränderlicher Parameter ist : so ist der Differentialquotient $\frac{d y}{d x}$ ebenfalls eine Function von $\alpha, x$ und $y$. Giebt man nun den Coordinaten $x$ und $y$ beliebige, aber constante Werthe, so kann man aus der Gleichung $f(x, y, \alpha)=0$, wenn dieselbe algebraisch und vorn $n$ ten Grade ist, $n$ Werthe des $\alpha$ finden. Diese sodann in dem Ausdrucke des ersten Differentialquotienten substituirt, giebt $n$ Werthe desselben; d. h. durch einen in der Ebene des Systems beliebig gewählten Punct gehen immer $n$ Aeste (reale oder imaginäre) hindurch. Dass $n$ durch einen Punct gehende Aeste sich rechtwinklig durchschneiden, hat keinen Sinn; die Bedeutung passt nur für zwei derselben, oder auch für mehrere Paare unter sich. Es seien also $\alpha_{1}, \alpha_{2}, \alpha_{3}, \ldots \alpha_{n}$ die $n$ Wurzeln der Gleichung $f(x, y, \alpha)=0$, so lässt sich dieselbe auf die Form 
$\left(\alpha-\alpha_{1}\right)\left(\alpha-\alpha_{2}\right)\left(\alpha-\alpha_{3}\right) \ldots\left(\alpha-\alpha_{n}\right)=0$ bringen, und wenn nun $\alpha_{1}$ und $\alpha_{5}$ zwei Werthe des $\alpha$ sind, für welche die zugehörigen, in dem Puncte $x, y$ sich schneidenden Aeste rechtwinklig auf einander stehen sollen, so kann man von den übrigen Factoren der Gleichung $f(x, y, \alpha)=0$ ganz absehen und nur die Gleichupg $(\alpha-\alpha)\left(\alpha-\alpha_{s}\right)=0$ in Betracht ziehen: das heisst, man hat es überall nur mit Systemen von Curven zu thun, welche in Beziehung auf den veränderlichen Párameter vom zweiten Grade sind. Dieselben können freilich, wenn sie dazu vorbereitet sind, in Beziehung auf $x$ und $y$ alle die Irrationalitäten enthalten, welche die Auflösung von Gleichungen höherer Grade mit sich führt, und wenn sie sodann rational gemacht werden, kann auch der Parameter $\alpha$ in ihnen zu beliebig hohen Graden aufsteigen. Die allgemeinste zu untersuchende Form der in Rede stehenden Systeme wird also $u \alpha^{2}+v a+w=0$ sein, wo $u, v, w$ Functionen von $x$ und $y$ sind; dividirt man aber durch $u$ und setzt $\frac{v}{u}=2 z, \frac{w}{u}=-z_{1}^{2}$ (die Form eines negativen Quadrates für dieses letzte Glied erweisèt sich in dem Folgenden als die passendste), wo $z$ und $z_{1}$ wieder Functionen von $x$ und $y$ sein können, so erhält man

$$
\text { 1. } \alpha^{2}+2 \alpha z-z_{1}^{2}=0
$$

für die weiter zu behandelnde Gleichung des Systems, in welcher $z$ und $z_{1}$ die zu bestimmenden Grössen sind. Setzt man, nach der gewohnten Art der Bezeichnung der partiellen Differentialquotienten, $d z=p d x+q d y$ und $d z_{1}$ $=p_{1} d x+q_{1} d y$, so giebt die Differentiation in Beziehung auf $x$ und $y$ :

also

$$
a(p d x+q d y)-z_{1}\left(p_{1} d x+q_{1} d y\right)=0,
$$

$$
\text { 2. } \quad \frac{d y}{d x}==\frac{p \alpha-p_{1} z_{1}}{q \alpha-q_{1} z_{1}} \text {. }
$$

Die Bedingung des rechtwinkligen Schneidens der beiden durch den Punct $x, y$ gehenden Aeste des Systems ist nun die, dass das Product der beiden Differentialquotienten; welche den beiden Werthen des $\alpha$ angehören, gleich -1 sei. 'Also hat man, wenn die beiden aus der Gleichung (1.) zu entnehmenden Werthe von $\alpha_{1}$ durch $\alpha_{1}$ und $\alpha_{2}$ bezeichnet werden:

oder, entwickelt,

$$
\left(\frac{p \alpha_{1}-p_{1} z_{1}}{q \alpha_{1}-q_{1} z_{1}}\right) \cdot\left(\frac{p \alpha_{2}-p_{1} z_{1}}{q \alpha_{2}-q_{1} z_{1}}\right)=-1
$$

$$
\left(p^{2}+q^{2}\right) b_{1} \alpha_{2}-z_{1}\left(p p_{1}+q q_{1}\right)\left(\alpha_{1}+\alpha_{2}\right)+\left(p_{1}^{2}+q_{2}^{2}\right) z_{1}^{2}=0
$$


und da aus der quadratischen Gleichung (1.) $\alpha_{1} \alpha_{2}=-z_{1}^{2}, \alpha_{1}+\alpha_{2}=-2 z$ folgt; so erhält man, nach Weglassung des gemeinschaftlichen Factors $z_{1}$ :

$$
\text { 3. } \quad z_{1}\left(p^{2}+q^{2}-p_{1}^{2}-q_{1}^{2}\right)-2 z\left(p p_{1}+q q_{1}\right)=0 \text {. }
$$

Die Bestimmung von $z$ und $z_{1}$, und mit ihr die vollständige Lösung der Aufgabe, hangt also von der Integration dieser partiellen Differentialgleichung von vier Variabeln, den zwei abhängigen $z$ und $z_{1}$ und den zwei unabhängigen $x$ und $y$ ab.

Die allgemeine Integration der Gleichung (3.) lässt sich enweder gar nicht, oder doch sicherlich nur in einer Form ausführen, welche dem Zwecke, einfache Systeme von Curven zu finden, nicht entsprechen würde: Dies zeigt sich schon darin, dass das vollständige Integral, wie leicht zu sehen, eine unendliche Anzahl willkürlicher Functionen enthalten müsste. Wir verzichten deshalb auf die vollständige Allgemeinheit und suchen nur einfache, wenn auch besondere Auflösungen der Differentialgleichung.

Zu diesem Zwecke bietet sich zunächst das Mittel dar, der Gleichung (3.) durch folgende zwei zu entsprechen:

$$
\text { 4. } p^{2}+q^{2}-p_{1}^{2}-q_{1}^{2}=\mathbf{0} \text { und } p p_{1}+q q_{1}=\mathbf{0} \text {. }
$$

Aus der Verbindung dieser beiden Gleichungen folgt, wenn $q_{1}$ eliminirt wird: $q^{2}=p_{1}^{2}$, also

$$
p_{1}= \pm q \text { und } q_{1}=\mp p \text {. }
$$

Nennt man nun, wie gewöhnlich, $r, s, t$ die drei zweiten partiellen Differentialquotienten des $z$, und $r_{1}, s_{1}, t_{1}$ die des $z_{1}$, so geben diese beiden Gleichungen, wenn die erste nach $y$, die andere nach $x$ differentiirt wird:

$$
s_{1}=\mp t \text { und } s_{1}=\mp r \text {, also } r+t=0 \text {. }
$$

Das vollständige Integral dieser Gleichung ist bekanntlich

$$
\text { 5. } z=f(x+i y)+f(x-i y)-i F(x+i y)+i F(x-i y) \text {, }
$$

wo $i=V-1$. Hieraus folgt dann, vermittelst der Gleichung $p=\mp q_{1}$, der Werth des $z_{1}$, nämlich:

$$
\text { 6. } \quad z_{1}=i f(x+i y)-i f(x-i y)+F(x+i y)+F(x-i y) \text {; }
$$

wo das doppelte Vorzeichen, auf welches hier nichts ankommt, weggelassen ist. Diese Werthe des $z$ und $z_{1}$, welche den beiden Gleichungen (4.) und folglich auch der Gleichung (3.) genügen, geben, in der Gleichung $a^{2}+2 a z-z_{1}^{2}=0$ substituirt, die erste allgemeine Formel für die gesuchten Systeme einander rechtwinklig schneidender Curven. Bestimmt man die beiden willkürlichen Functionen so, dass $f(x+i y)=\frac{1}{2}(x+i y)$ und $F(x+i y)=0$ ist, so erhält man, als speciellen Fall: 


$$
\alpha^{2}+2 a x-y^{2}=0
$$

welches das System der confocalen Parabeln ist. Nimmt man, etwas allgemeiner, $f(x+i y)=\frac{1}{2} a(x+i y)^{m}$ und $F\left(x+i y=\frac{1}{2} b(x+i y)^{n}\right.$ und führt die Polarcoordinaten, $x=\rho \cos \varphi, y=\rho \sin \varphi$ ein, so erhält man folgende Gleichung:

7. $\alpha^{2}+2 a\left(a \rho^{m} \cos m \varphi+b \rho^{n} \sin n \varphi\right)-\left(a \rho^{m} \sin m \varphi-b \rho^{n} \cos n \varphi\right)^{2}=0$.

Wir wollen uns auf eine nähere Discussion der durch diese Formel ausgedrückten Curven, welche, je nachdem $m$ und $n$ ganze oder gebrochene, positive oder negative Zahlen sind, stets einen andern Character haben, nicht einlassen, sondern gehen zur Integration der Gleichung (3.) zurück.

Das bisher angewendete Integrations-Verfahren lässt sich nämlich erweitern, dadurch, dass $z$ als Function von $z_{1}$ und einer neuen, später passend zu bestimmenden Variabel $z_{2}$ angenommen wird. Wenn $d z_{2}=p_{2} d x+q_{2} d y$ gesetzt wird, so erhält man durch Differentiation nach $x$ und nach $y$ :

$$
p=\frac{\partial z}{\partial z_{1}} p_{1}+\frac{\partial z}{\partial z_{2}} p_{2}, \quad q=\frac{\partial z}{\partial z_{1}} q_{1}+\frac{\partial z}{\partial z_{2}} q_{2}
$$

Diese Werthe von $p$ und $q$ in der Gleichung (3.) substituirt, geben

$$
\begin{gathered}
\left(z_{1}\left(\frac{\partial z}{\partial z_{1}}\right)^{2}-z_{1}-2 z \frac{\partial z}{\partial z_{1}}\right)\left(p_{1}^{2}+q_{1}^{2}\right)+z_{1}\left(\frac{\partial z}{\partial z_{2}}\right)^{2}\left(p_{2}^{2}+q_{2}^{2}\right) \\
+2\left(z_{1} \frac{\partial z}{\partial z_{1}} \frac{\partial z}{\partial z_{1}}-z \frac{\partial z}{\partial z_{2}}\right)\left(p_{1} p_{2}+q_{1} q_{2}\right)=0 .
\end{gathered}
$$

Bestimmt man jetzt die beiden Variabeln $z_{1}$ und $z_{2}$ durch die Gleichungen $p_{1}^{2}+q_{1}^{2}-p_{2}^{2}-q_{2}^{2}=0$ und $p_{1} p_{2}+q_{1} q_{2}=0$, deren vollständige Lösung gefunden ist, so geht diese Gleichung in

$$
\text { 8. } \quad z_{1}\left(\left(\frac{\partial z}{\partial z_{1}}\right)^{2}+\left(\frac{\partial z}{\partial z_{2}}\right)^{2}-1\right)-2 z \frac{\partial z}{\partial z_{1}}=0
$$

über. Ein particuläres Integral derselben, welches sich von selbst ergiebt, ist

$$
2 z=1-z_{1}^{2}-z_{2}^{2}
$$

Dieses giebt sogleich folgende neue allgemeine Formel für Systeme rechtwinklig einander sich schneidender Curven:

$$
\alpha^{2}+\alpha\left(1-z_{1}^{2}-z_{2}^{2}\right)-z_{1}^{2}=0,
$$

welcher auch folgende Form gegeben werden kann:

$$
\text { 9. } \frac{z_{1}^{2}}{\alpha}+\frac{z_{2}^{2}}{\alpha+1}=1 \text {; }
$$

พо

$$
\begin{aligned}
& z_{1}=f(x+i y)+f(x-i y)-i F(x+i y)+i F(x-i y), \\
& z_{2}=i f(x+i y)-i f(x-i y)+F(x+i y)+F(x-y) .
\end{aligned}
$$

Der einfachste besondere Fall dieser Formel, nämlich wenn $f(x+i y)=\frac{1}{2}(x+i y)$ 
und $F(x+i y)=0$ angenommen wird, giebt das System der Kegelschnitte mit zwei gemeinschaftlichen Brennpuncten, nämlich:

$$
\frac{x^{2}}{\alpha}+\frac{y^{2}}{\alpha+1}=1 \text {. }
$$

Setzt man ferner, wie oben, etwas allgemeiner, $f(x+i y)=\frac{1}{2} a(x+i y)^{m}$ und $F(x+i y)=\frac{1}{2} b(x+i y)^{n}$, und führt Polarcoordinaten ein, so erhält man folgende Gleichung:

$$
\text { 10. } \quad \frac{\left(a \varrho^{m} \cos m \varphi+b Q^{n} \sin n \varphi\right)^{2}}{\alpha}+\frac{\left(a \rho^{m} \sin m \varphi+b \varrho^{n} \sin n \varphi\right)^{2}}{\alpha+1}=1 \text {, }
$$

welche eine sehr reiche Auswahl einfacher und interessanter Curven be zeichnet.

Die partielle Differentialgleichung (8.) lässt sich auch allgemein integriren, und liefert dann eine noch weit allgemeinere, drei willkürliche Functionen enthaltende Formel für die gesuchten Systeme von Curven. Man findet durch die bekannten Methoden das Integral der Gleichung (8.), als Resultat der Elimination der Grösse $c$, aus folgenden beiden Gleichungen:

$$
\text { 11. }\left\{\begin{array}{c}
2 c z-c^{2} z_{1}^{2}-\left(c z_{2}+\varphi(c)\right)^{2}+1=0 \text { und } \\
z-c z_{1}^{2}-\left(c z_{2}+\varphi(c)\right)\left(z_{2}+\varphi^{\prime}(c)\right)=0
\end{array}\right.
$$

wo $\varphi(c)$ die willkürliche Function und $\varphi^{\prime}(c)$ der erste Differentialquotient derselben ist. Wird der aus der zweiten dieser Gleichungen entnommene Werth von $z$ in der Gleichung $\alpha^{2}+2 \alpha z-z_{k}^{2}=0$ substituirt, ond ausserdem $z$ aus den beiden Gleichungen $(11 /)$ eliminirt, so erhält man

$$
\text { 12. }\left\{\begin{array}{l}
\alpha^{2}+2 \alpha\left(c z_{1}^{2}+c z_{2}+\varphi(c)\right)\left(z_{2}+\varphi^{\prime}(c)\right)-z_{1}^{2}=0 \\
c^{2} z_{1}^{2}+\left(c z_{2}+\varphi(c)\right)\left(c z_{2}+2 c \varphi^{\prime}(c)-\varphi(c)\right)+1=0
\end{array}\right.
$$

Diese beiden Gleichungen enthalten die gesuchte Formel mit drei willkürlichen Functionen, wenn nämlich $c$ eliminirt wird und für $z_{1}$ und $z_{2}$ die bei (9.) angegebenen, Werthe gesetzt werden. Auf äbnliche Weise lassen sich noch viele andere, mehr oder weniger complicirte, Integrationen der Differentialgleichung (3.) ausführen und dadurch allgemeine Formeln für Systeme sich selbst rechtwinklig schneidender Curven friden. Wir wollen diese Formeln nicht hăuen, sondern in dieser Beziehung nur ene allgemeine Bemerkung macheh, zu welcher die beiden Fordiln

$$
\alpha^{2}+2 \alpha z_{1}-z_{2}^{2}=0 \text { und } \frac{z_{1}^{2}}{\alpha}+\frac{z_{2}^{2}}{\alpha+1}=1 \text { (1) }
$$

Anlass geben: Dieselben entstehen aus den bekaninten beiden Systemén ron confocalen Kegelschnitten, nämlich: 
an $a^{2}+2 a x-y^{2}=0$ und $\frac{x^{2}}{\alpha}+\frac{y^{2}}{\alpha+1}=1$

unmittelbar, wenn $z_{1}$ statt $x$ und $z_{2}$ statt $y$ gesetzt wird. Im Allgemeinen lässt sich nun leicht zeigen, dass, wenn in der die Coordinaten $x$ und $y$ enthaltenden Gleichung irgend eines Systems sich selbst rechtwinklig sohneidender Curven, $z_{1}$, statt $x$ und $z_{z}$ statt $y$ gesetzt 'wird; dadurch wieder die Gleichung eines ebenso beschaffenen Systems hervorgeht. Es sei nämlich; wie' obén! $d z_{1}=p_{1} d x+q_{1} d y$ und $d z_{2}=p_{2} d x+q_{2} d y$; ferner seien $u$ und $u_{1}$ zwei Functionen von $x$ und $y$, welche machen, dass $\alpha^{2}+2 \alpha u-u_{1}^{2} \doteq 0$ die Gleichung' eines der sich selbst rechtwinklig schneidenden Systeme von Curven ist: so muss, wie wir oben sahen, folgende Gleichung Statt haben:

$$
\begin{array}{ll}
\text { 13. } & u_{1}\left(\left(\frac{\partial u}{\partial x}\right)^{2}+\left(\frac{\partial u}{\partial y}\right)^{2}-\left(\frac{\partial u_{1}}{\partial x}\right)^{2}-\left(\frac{\partial u_{1}}{\partial y}\right)^{2}\right) \\
& -2 u\left(\frac{\partial u}{\partial x} \frac{\partial u_{1}}{\partial x}+\frac{\partial u}{\partial y} \cdot \frac{\partial u_{1}}{\partial y}\right)=0
\end{array}
$$

und umgekehrt: wenn diese Gleichung befriedigt ist, so ist $\alpha^{2}+2 \alpha u-u_{1}^{2}=0$ die Gleichung eines solchen Systems. Betrachtet man nun $u$ und $u_{1}$ als Functionen von $z_{1}$ und $z_{1}$, welche wieder die bekannten, durch die. Gleichungen bei (9.) bestimmten Functionen von $x$ und $y$ sind, so erhält man durch Differenitiation:

$$
\begin{array}{ll}
\frac{\partial u}{\partial x}=\frac{\partial u}{\partial z_{1}} p_{1}+\frac{\partial u}{\partial z_{2}} p_{2}, & \frac{\partial u}{\partial y}=\frac{\partial u}{\partial z_{1}} q_{1}+\frac{\partial u}{\partial z_{2}} q_{2}, \\
\frac{\partial u_{1}}{\partial x}=\frac{\partial u_{1}}{\partial z_{1}} p_{1}+\frac{\partial u_{1}}{\partial z_{2}} p_{2}, & \frac{\partial u_{1}}{\partial y}=\frac{\partial u_{1}}{\partial z_{1}} q_{1}+\frac{\partial u_{1}}{\partial z_{2}} q_{2} .
\end{array}
$$

Substituirt man diese Werthe der nach $x$ und $y$ genommenen Differentialquotienten in der Gleichung (13.), und macht von den beiden Gleichungen $p_{1}^{2}+q_{1}^{2}-p_{2}^{2}-q_{2}^{2}=0$ und $p_{1} p_{2}+q_{1} q_{2}=0$ Gebrauch, so erhält man die Gleichung:

$$
\text { 14. } \begin{aligned}
u_{1} & \left(\left(\frac{\partial u}{\partial z_{1}}\right)^{2}+\left(\frac{\partial u}{\partial z_{2}}\right)^{2}-\left(\frac{\partial u_{1}}{\partial z_{1}}\right)^{2}-\left(\frac{\partial u_{1}}{\partial z_{2}}\right)^{2}\right) \\
& -2 u\left(\frac{\partial u}{\partial z_{1}} \frac{\partial u_{1}}{\partial z_{1}}+\frac{\partial u}{\partial z_{2}} \frac{\partial u_{1}}{\partial z_{2}}\right)=0,
\end{aligned}
$$

welche sich von der Gleichung (13.) nur dadurch unterscheidet, dass statt $x$ und $y$ hier $z_{1}$ und $z_{2}$ steht. Mit der Gleichung (13.) wird nun allemal diese Gleichung (14.) befriedigt; woraus die Richtigkeit der obigen Bemerkung klar ist.

Nachdem wir hinlänglich die Mittel gezeigt haben, wie man Systeme einander überall rechtwinklig schneidender Curven finden kann, wollen 
wir noch eine allgemeine Eigenschaft dieser Systeme nachweisen, nämlich die, dass sie immer eine bestimmte Anzzabl von Brennpuncten haben, welche allen Curven eines Systems gemeinsam sind. Bekanntlich hat Pbücker zuerst eine allgemeine Definition für die Brènpuncte der Curven beliebiger Ordnungen gegeben, welcher zufolge sie diejenigen Puncte in der'Ebene einer Curve sind, von denen aus sich zwei imaginäre Tangenten an die Curve ziełhen lassën, welche mit der Abscissenaxe (also mit einer beliebigen Graden) Witikel bilden, deren trigonometrische Tangenten die Werthe $+V-1$ und $-V-1$ háben. Für unsern gegenwärtigen $Z_{\text {weck, }}$, wo es sich nur um die allèn Curven einès Systems gemeinsamen Brennpuncte handelt, werden wir' zwar auf eine andere Weise zu den Brennpuncten gelangen, aber dann nachweisen, dass dieselben auch in dem allgemeinen Sinne Brennpuncte sind, welchen Plücker diesen Puncten treffend gegeben hat.

In einem Systeme von Curven, welche einander überall rechtwinklig schneiden, dürfen zwei unendlich nahe Curven durchaus nicht reale Durchschnittspuncte haben: denn wären dergleichen vorhanden, so würden in ihnen die sich schneidenden unendlich nahen Curven nicht einen rechten, sandern einen verschwindend kleinen Winkel bilden. Das System darf darum keine reale Grenzcurve haben. Sucht man nun die Grenzcurve unseres Systems, so erhält man durch Elimination des $\alpha$ aus den beiden Gleichungen

$$
\alpha^{2}+2 \alpha z-z_{1}^{2}=0 \text { und } \alpha+z=0 \text {, }
$$

folgende Gleichung der Grenzcurve:

$$
\left.z^{2}+z_{1}^{2}=0^{*}\right)
$$

welche wirklich, weil wir dem dritten Gliede unserer allgemeinen Gleichung die Form eines negativen Quadrats gaben, wenn $z$ und $z_{1}$ nicht etwa einen gemeinschaftlichen Factor haben, eine reale Linie nicht giebt. Die imaginären Aeste derselben aber haben reale Durchschnittspuncte, welche durch die beiden Gleichungen

$$
z=0 \text { und } z_{1}=0
$$

bestimmt werden und welche eben die für alle Curven des Systems constanten Brennpuncte sind, um welche es sich handelt. Um nun nachzuweisen, dass dieselben auch nach der Definition von Plücker Brennpuncte sind, betrachte ich zwei Curven des Systems, nämlich

*) Es ist kaum nöthig, zu erinnern, dass hier $z$ und $z_{1}$ nicht die beschränkte, durch die Gleichungen (5.) und (6.) angegebene Bedeulung haben, sondern dass es irgend Functionen von $x$ und $y$ sind, welche der Gleichung ( 3 ) genügen. 


$$
\alpha^{2}+2 \alpha z-z_{1}^{2}=0 \text { und } \beta^{2}+2 \beta z-z_{1}^{2}=0 .
$$

Die respectiven Differentialquotienten für dieselben sind

$$
\frac{d y}{d x}=-\frac{p \alpha-p_{1} z_{1}}{q \alpha-q_{1} z_{1}} \quad \text { und } \quad \frac{d y}{d x}=-\frac{p \beta-p_{1} z_{1}}{q \beta-q_{1} z_{1}} \text {. }
$$

Für diejenigen Puncte nun, in welchen diese beiden Curven sich schneiden, es geschehe real oder imaginair, ist das Product dieser Differentialquotienten gleich - 1: nimmt man also $\beta$ unendlich wenig verschieden von $\alpha$ an, so werden die beiden Differentialquotienten einander gleich, also das Product derselben zu einem Quadrate, und man erhält

$$
\left(\frac{d y}{d x}\right)^{2}=-1 \text { und } \frac{d y}{d x}= \pm V-1
$$

für alle Puncte, in welchen zwei unendlich nahe Curven eines Systems sich schneiden, mithin auch für alle Puncte der Grenzcurve. Legt man nun Tangenten an die Grenzcurve, in den durch die Gleichungen $z=0$ und $z_{1}=0$ bestimmten Puncten, so sind dieselben zugleich Tangenten gewisser Curven des Systems: die von ihnen mit der Abscissenaxe gebildeten Winkel haben die trigonometrischen Tangenten $\pm V-1$; sie sind also Brennpuncte für diese bestimmten Curven des Systems, und da dieses Resultat von a ganz unabhängig ist, so sind es gemeinsame Brennpuncte für alle Curven des Systems.

Breslau im September 1847. 